\title{
Metastatic Spread Of Oral Squamous Cell Carcinoma
}

\section{To Level 2b: A Retrospective Study}

\section{Sarwar M, Walker DT, Barrett B, Gulati A}

Queen Victoria Hospital, East Grinstead

\begin{abstract}
INTRODUCTION
Due to the high levels of occult lymph node metastasis in oral cavity squamous cell carcinoma, the vast majority of patients undergo selective neck dissection levels 1-4. Level 2b nodal metastasis, however, is uncommon. The current standard of treatment is dissection of level $2 b$ as part of treatment for cancer but this has comorbidity associated with it which includes spinal accessory nerve dysfunction and related shoulder pain and disability.
\end{abstract}

\section{AIMS}

1. To describe the rate of metastasis to $2 \mathrm{~b}$ from oral cavity squamous cell carcinoma

2. Identify specific subsites, features of the primary and patient factors leading to higher metastasis rate

3. Review recommendations for $2 b$ dissection in all oral cavity carcinoma

\section{METHOD}

A retrospective cohort study on all neck dissections performed as part of treatment for OSCC over a period of 5 years from 2013 -2017. All neck dissections done at this centre are pinned out by the surgeon post operatively onto a standardised laminated template, with 2b identified by a silk stitch intraoperatively. Details regarding the primary tumour characteristics, lymph node dissection features and nodal distribution were collected and tabulated. Specific metastases to $2 b$ could be identified in most reports given the intraoperative suture labelling.
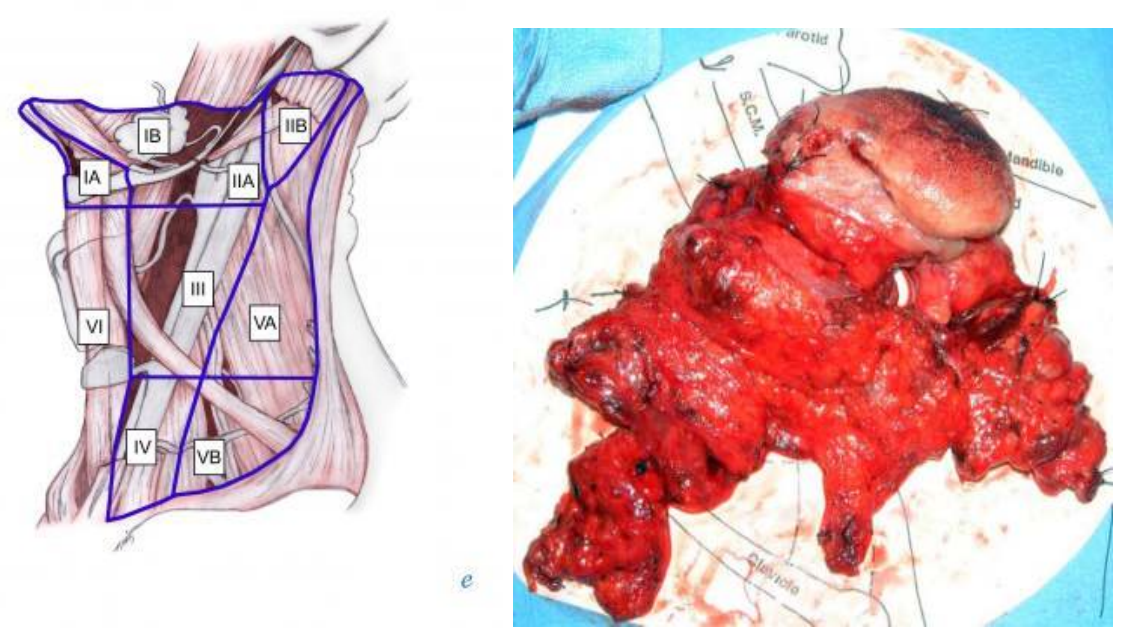

RESULTS

- 348 neck dissections for oral cavity squamous cell carcinoma were recorded

- 135 patients were found to have metastasis to the neck (38.8 \% of total)

- 9 patients had metastasis to level $2 \mathrm{~b}(6.7 \%)$ alone

- 48 patients were found to have disease spread to level 2 ( $2 \mathrm{~b}$ indistinguishable)

- $44.4 \%$ of patients with metastasis to level $2 \mathrm{~b}$ had primary SCC of lateral tongue

- A further major contribution to this group also came from retromolar trigone SCC (33.3\%)

- Higher T Stage, older age at diagnosis, and poor differentiation were all significant risk factors for metastasis to $2 b$

- Primaries with a cohesive front were less likely to metastasise to this zone

\section{CONCLUSIONS}

- Our results support current evidence that the incidence of metastasis to level $2 \mathrm{~b}$ from oral cavity squamous cell carcinoma remains low

- Incidence is higher with lateral tongue primary, high T Stage and poorly differentiated primary. Super selective neck dissection should not be employed in such cases

- We have not identified any subgroups for whom the risk of $2 \mathrm{~b}$ metastasis is significantly low enough to recommend limiting neck dissection in this area 